\title{
Highly Sensitive Filter Paper Substrate for SERS Trace Explosives Detection
}

\author{
Pedro M. Fierro-Mercado and Samuel P. Hernández-Rivera \\ Department of Chemistry, ALERT-DHS Center of Excellence, Center for Chemical Sensors Development, \\ University of Puerto Rico at Mayagüez, P.O. Box 9000, Mayagüez, PR 00681-9000, USA \\ Correspondence should be addressed to Samuel P. Hernández-Rivera, samuel.hernandez3@upr.edu
}

Received 17 March 2012; Accepted 4 September 2012

Academic Editor: Keith Carron

Copyright () 2012 P. M. Fierro-Mercado and S. P. Hernández-Rivera. This is an open access article distributed under the Creative Commons Attribution License, which permits unrestricted use, distribution, and reproduction in any medium, provided the original work is properly cited.

\begin{abstract}
We report on a novel and extremely low-cost surface-enhanced Raman spectroscopy (SERS) substrate fabricated depositing gold nanoparticles on common lab filter paper using thermal inkjet technology. The paper-based substrate combines all advantages of other plasmonic structures fabricated by more elaborate techniques with the dynamic flexibility given by the inherent nature of the paper for an efficient sample collection, robustness, and stability. We describe the fabrication, characterization, and SERS activity of our substrate using 2,4,6-trinitrotoluene, 2,4-dinitrotoluene, and 1,3,5-trinitrobenzene as analytes. The paper-based SERS substrates presented a high sensitivity and excellent reproducibility for analytes employed, demonstrating a direct application in forensic science and homeland security.
\end{abstract}

\section{Introduction}

In last decade, the potential exposure of civilian and military populations to hazardous materials (chemical, biological, and highly energetic) has attracted attention of authorities, because it has become an increasingly likely scenario. The rapid and accurate detection and identification of possible threats is crucial to neutralize potentially threatening situations. In order to detect and identify these hazards, the ideal technology should exhibit high sensitivity and reproducibility in analyte detection, require little or no sample preparation, and be robust, easy to use, and portable for field operations [1].

A series of analytical methodologies, principally chromatographic and spectrometric techniques, are routinely carried out in specialized laboratories using standard methods to assess chemical and biological hazards. These usually require several steps including sampling, handling, preparation, and analysis, which make the entire process timeconsuming and difficult to apply in the field.

Raman spectroscopy is a well-suited technique for the identification, characterization, and quantification of unknown targets [2-5]. Raman spectroscopy is particularly advantageous because the technique does not present interferences from water (which is the universal solvent), requires minor or no sample preparation, and produces vibrational information useful as unique spectral signature of the analyte of interest. In addition, the miniaturization of Raman equipment has added robustness to the technique, which has made possible its use in different environments outside the laboratory confinement. However, development of Raman spectroscopy as analytical technique for trace detection of hazardous materials has not been possible, mostly due to the extremely low Raman scattering cross sections characteristic from these compounds.

Discovery of surface-enhanced Raman scattering (SERS) in mid of 1970s and its application in spectroscopic analysis has emerged as a tool that overcomes limitation of normal Raman scattering by greatly enhancing Raman signals intensities [6-13]. It is common to observe SERS enhancements from 6 to 8 orders of magnitude, although under ideal conditions it is possible to detect a single molecule [14, 15]. The mechanisms by which Raman enhancement in SERS is possible even remain unclear, but the scientific community 
attributes the phenomenon to two main mechanisms: a coupling of the electromagnetic field of the laser radiation with plasmonic oscillations of electrons on the metal nanostructured surfaces and the chemisorption of the analyte on metal surfaces with exchange of electron density and bond formation. Thus, SERS appears as a powerful technique for trace level detection of chemical and biological species with a great impact in life and environmental sciences and defense and homeland security.

Intense efforts have been intended to translate the capabilities of SERS to viable sensing systems that can be utilized for routine analysis in the lab or in the field. Metallic colloidal suspensions were the first and simplest SERS substrates employed due to ease of preparation and large Raman enhancements [16]. However, relatively high instability, poor reproducibility, and the requirement of sampling in the liquid state render these systems impractical for many applications. Continued researches have allowed the development of methodologies to produce substrates with ordered and uniform nanostructures of different materials, highly reproducible and with large SERS signals intensity enhancements. These include nanolithography and metal film over nanostructures (MFON) fabrications [17-21]. In the first one, a surface (mostly polymeric) is nanostructured using electron-beam lithography (EBL). Later a layer of metal is deposited using chemical vapor deposition (CVD) generating a homogeneous surface with a variety of shapes, sizes, and orientations. The MFON substrates can be fabricated by depositing a layer of silica or polystyrene nanoparticles onto a rigid plate and then these nanoparticles are overcoated with a metal film, particularly silver or gold, creating substrate architectures with improved stability. Despite the great benefits of the above-mentioned techniques, fabrication procedure is time consuming, requiring large efforts and skills of fabrication, and the overall process is not cost efficient, resulting in substrates presenting poor sample collection efficiencies due to rigid design restrictions.

In this paper, a new methodology is described for preparing reproducible and highly sensitive SERS-active substrates using commercial laboratory filter paper (Whatman filter paper grade 1) as support for deposition of gold nanoparticles by means of thermal inkjet technology (TIJ). The novel SERS sensing platforms are extremely inexpensive, highly practical, and efficient in collection of samples by merely swabbing a contaminated surface or surfaces that are believed to have been in contact with hazardous materials. Actually, a swabbing method is the strategy employed for passenger screening at airport portals using ion mobility spectrometry as highly energetic materials (HEM) detection technique. Our paper substrate demonstrates high sensitivity and reproducibility in detection of HEM such as trinitrotoluene (TNT) and some of its degradation products. A few reports of SERS substrates prepared using metal-coated paper have been published in the literature [22-24], but to the best of our knowledge, the study presented here represents the first SERS substrate over filter paper with clear applicability in the detection of trace level of HEM, highly flexible and efficient in sample collection from contaminated surfaces.

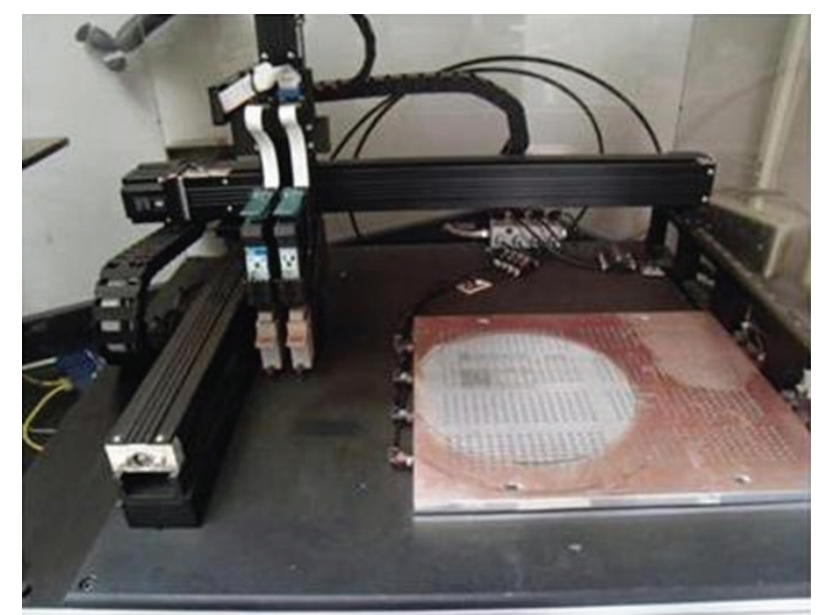

FIgURe 1: Photograph of thermal inkjet system used for preparing SERS substrates.

\section{Experimental Section}

2.1. Materials. Hydrogen tetrachloroaurate $\left(\mathrm{HAuCl}_{4} \cdot 3.5 \mathrm{H}_{2} \mathrm{O}\right.$; 99.99\%) from Strem Chemicals (Newburyport, MA) was the metal precursor material for the colloidal gold. Reagentgrade ethylene glycol, polyvinylpyrrolidone (PVP, K30, M 40,000 ), sodium bicarbonate, acetone, and isopropyl alcohol were purchased from Fisher Scientific, Inc. (Chicago, IL). HEM 2,4,6-trinitrotoluene (TNT), 2,4-dinitrotoluene (2,4$\mathrm{DNT}$ ), and 1,3,5-trinitrobenzene (TNB) were acquired as GC/MS grade standards from ChemService, Inc. (West Chester, PA). All chemicals were used as received.

2.2. Ink Preparation. The nanosized gold colloids used were synthesized by chemical reduction using the modified polyol method described by Lee et al. [25]. Briefly, a $50 \mathrm{~mL}$ two-neck round bottom flask was charged with $75 \mathrm{mg}$ of $\mathrm{NaHCO}_{3}$, $500 \mathrm{mg}$ of PVP, and $18 \mathrm{~mL}$ of ethylene glycol. The mixture was heated with reflux at $80^{\circ} \mathrm{C}$ for $1 \mathrm{~h}$ under continuous stirring. Then, $25 \mathrm{mg}$ of $\mathrm{HAuCl}_{4} \cdot 3.5 \mathrm{H}_{2} \mathrm{O}$ dissolved in $2.0 \mathrm{~mL}$ of ethylene glycol was quickly added and the mixture was left in reflux at $80^{\circ} \mathrm{C}$ for $2 \mathrm{~h}$. At the end of the reaction the colloidal dispersion was cooled until room temperature and the gold nanocrystals were separated from ethylene glycol after the addition of a large amount of acetone followed by centrifugation. Then, the precipitates were redispersed in isopropyl alcohol for characterization studies and thermal ink-jet depositions.

2.3. Printer Setup. The SERS-active substrates investigated in this study were fabricated using an ImTech Imaging System model I-Jet 312 S (ImTech, OR, USA), computer-controlled, designed as a prototype experimental model for printing on solid surfaces (Figure 1).

The nanoparticles ink was ejected using an HP 51645A print head supplied by General Technology Co., LTD (Tokyo, Japan). Printout of the substrates was achieved through a pattern designed using MS Paint software (Microsoft, Redmond, WA) on laboratory filter paper 
(Whatman Grade 1;11 $\mu \mathrm{m}$ ) using a resolution of $600 \times 600$ dots per inch (dpi). The uniform ejection of the droplets was performed by applying $12 \mathrm{~V}$ of print head voltage, $2.0 \mu \mathrm{s}$ of firing pulse at a maximum frequency of $12 \mathrm{KHz}$.

\section{Measurements and Analysis}

3.1. Characterization of SERS Substrates. Nanoparticle sizes were obtained from images obtained by a model 922 Zeiss High Resolution Transmission Electron Microscopy (HR-TEM; Carl Zeiss Microscopy, LLC, Thornwood, NY) operated at $200 \mathrm{kV}$. The UV-Vis spectra of the nanoparticle suspension were obtained using a UV-visible spectrometer (Agilent, Cary 5000 UV-Vis-NIR Spectrophotometer). Morphological images were taken using a JEOL model 5800LV scanning electron microscope (Peabody, MA) using an accelerating voltage of $15 \mathrm{keV}$.

3.2. SERS Measurements. The SERS spectra were obtained by using a portable Raman measurement system (Raman Systems, R-3000QE Raman spectrometer, Agiltron, Inc., Woburn, MA) equipped with a laser at $785 \mathrm{~nm}$ excitation wavelength and using a power of $19 \mathrm{~mW}$. A $5 \mathrm{~s}$ integration time was used for acquisition of the SERS spectra in the range from 200 to $1800 \mathrm{~cm}^{-1}$. For SERS assays, $5.0 \mu \mathrm{L}$ of a solution of HEM in ethanol was transferred to the SERS substrates using a micropipette, which immediately spread forming a circle of $\sim 1.0 \mathrm{~cm}$ of diameter. The substrates were then left in air to dry. SERS measurements were acquired in three different substrates contaminated with the analytes and at three different locations within each substrate, resulting in a total of nine SERS measurements per analyte concentration.

\section{Results and Discussion}

An important concern about the fabrication of SERS-active substrates is the possible overlap of analytical signal of interest from the analyte due to substrate signals. In this work, laboratory filter papers used in qualitative analytical techniques to determine and identify materials were used as substrates. These materials, which are essentially composed of cellulose, presented little or no interferences with signal of the analytes (HEM) studied.

Figure 2 shows the UV-Vis absorption spectra of the synthesized gold nanoparticles ( $\mathrm{Au} \mathrm{NP}$ ). The extinction spectrum clearly shows one well-resolved maxima at $526 \mathrm{~nm}$ attributed to the surface plasmon excitation of the essentially spherical $\mathrm{Au} \mathrm{NP}$. The synthesized quasispherical shape nanoparticles had an averaged diameter of $45 \mathrm{~nm}$ and appeared quite monodispersed as it can be seen in TEM image inset of the graph of Figure 2.

Figure 3 shows optical images of gold nanoparticles before and after being printed on paper. The fibrous morphology of the filter paper can be appreciated such that the cellulose nanofibers are braided into microfibers with variable diameters. Once the Au NP-loaded paper was dried, small agglomerates of nanoparticles irreversibly absorbed on the cellulose fibers could be observed. No noticeable changes in the Au density as detected by unaided eyes were observed

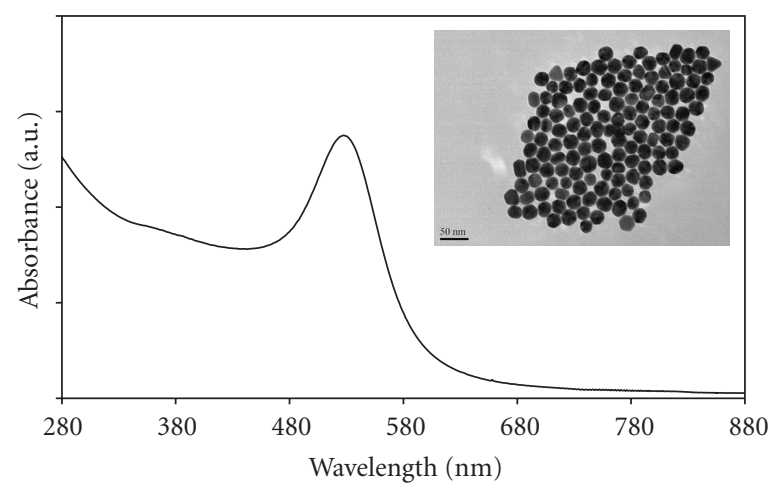

FigURE 2: UV-Vis adsorption spectra of Au NP suspension. Inset: TEM image of Au nanoparticles. Scale bar is $50 \mathrm{~nm}$.

after intense rinsing of the Au NP loaded paper with water or alcohol.

Scanning electron microscopy images reveal dense adsorption of the Au NP on the surface of the paper showing signs of aggregation (Figure 4(a)). High magnification SEM images show clusters of gold nanoparticles decorating fibers of the paper (Figure 4(b)). The surface density of the gold nanoclusters shown in Figure 4 assures substrates with high SERS activity. These clusters originate the localized surface plasmon resonance that in turn generates large SERS enhancement due to formation of hot spots.

This study is mainly concerned with the fabrication of SERS-active substrates as sensing platforms for the trace detection of HEM 2,4,6-trinitrotoluene (TNT) and some of its degradation products such as 2,4-dinitrotoluene (2,4DNT) and 1,3,5-trinitrobenzene (TNB). TNT is one of the most widely explosive used for both military and industrial applications. Its insensitivity to shock and friction makes it quite valuable and commonly is found in explosives blends to reduce the risk of accidental detonation of more sensitive high explosives [26].

It should be noted that the poor binding affinities of these species (nitroaromatic HEM) with metallic nanostructures, as well as the relative low Raman cross sections of these molecules and the catalytic and photochemical decomposition caused by intense laser exposure, make it difficult to obtain high quality SERS spectra from this class of compounds. For this reason, few studies related to these issues are found in the literature. Most of them deal with SERS studies of these species in aqueous solutions $[27,28]$.

Figure 5 shows normal Raman spectrum of solid TNT, 2,4-DNT, and 1,3,5-TNB obtained with the R-3000QE Raman $785 \mathrm{~nm}$ spectrometer. Raman shifts and relative intensities of Raman bands of TNT are in agreement with previously reported Raman spectra [29, 30].

Characteristic Raman bands for TNT include a very strong band at $1362 \mathrm{~cm}^{-1}$, and two bands at 1534 and $1617 \mathrm{~cm}^{-1}$ corresponding to symmetric and asymmetric $\mathrm{NO}_{2}$ stretching vibration, and aromatic $\mathrm{NO}_{2}$ conjugation, respectively. Similarly, bands at 1207 and $1085 \mathrm{~cm}^{-1}$ assigned to the ring breathing and $\mathrm{CH}$ (ring) bending modes, and the other peaks at 821 and $789 \mathrm{~cm}^{-1}$ corresponding to 


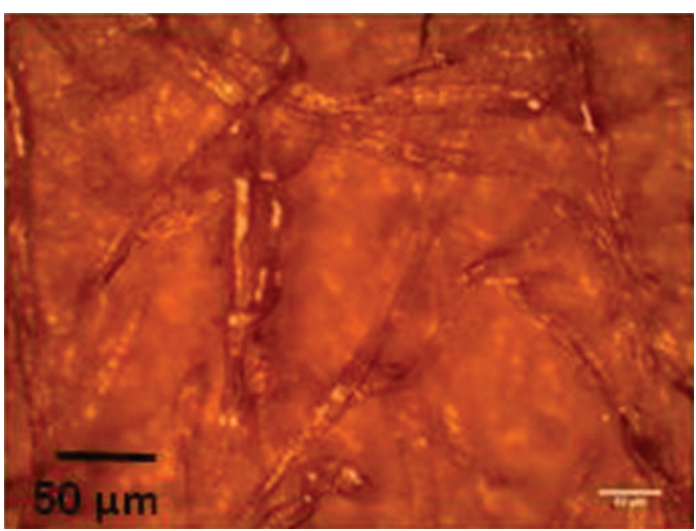

(a)

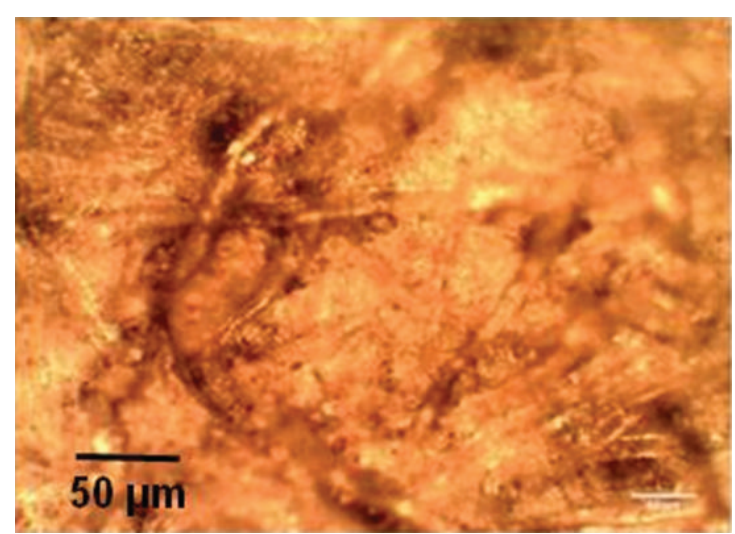

(b)

FIGURE 3: Optical images of (a) filter paper, and (b) inkjet-printed Ag NP on a Whatman grade 1 filter paper.

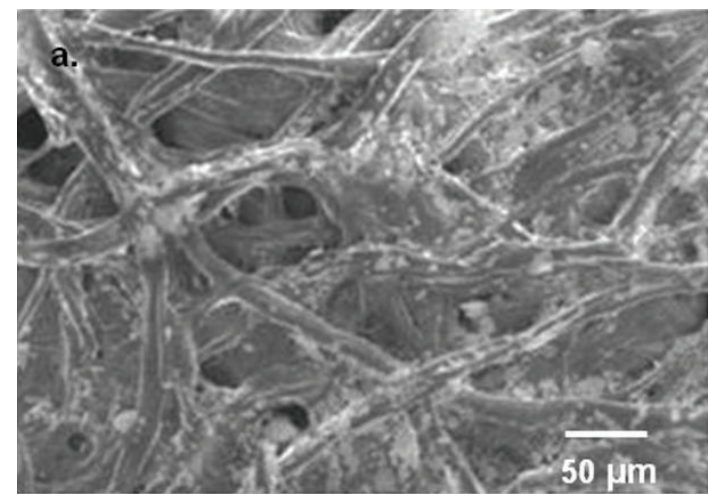

(a)

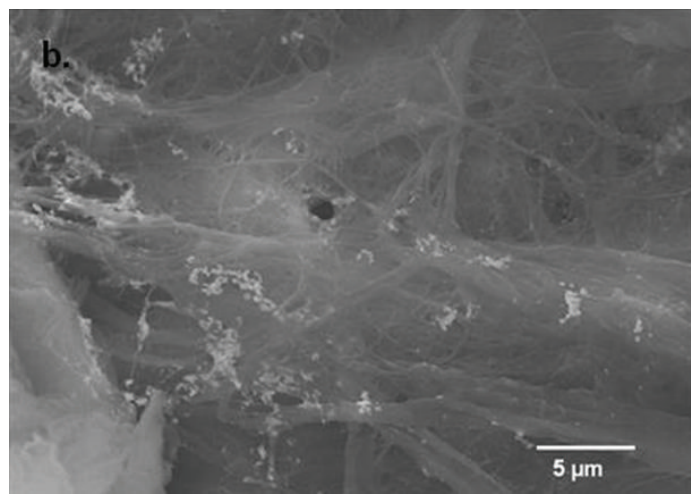

(b)

FIGURE 4: Scanning electron micrograph of printed Au NP on a Whatman grade 1 filter paper.

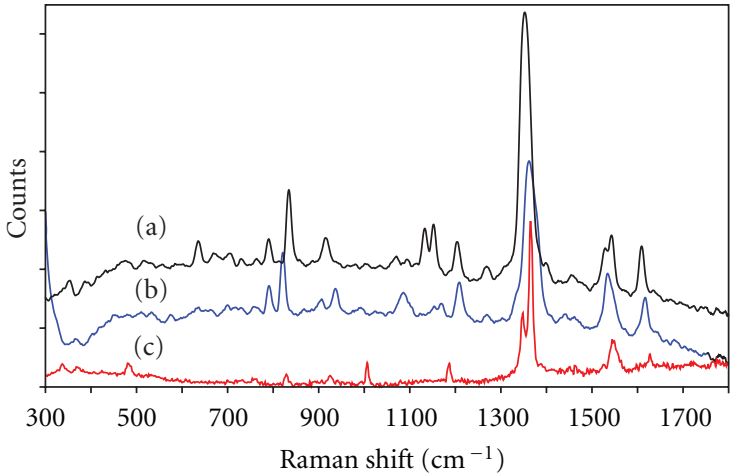

FIGURE 5: Normal Raman spectra of (a) 2,4-DNT, (b) TNT, and (c) $1,3,5-\mathrm{TNB}$.

scissoring $\mathrm{NO}_{2}$ mode and $\mathrm{CH}$ out-of-plane vibrations, can be appreciated.

The Raman spectrum of TNT adsorbed on our SERS substrate is a slightly different from that of bulk TNT (Figure 6(a)). Representative band at $1362 \mathrm{~cm}^{-1}$ is slightly shifted to lower wavenumbers, and an additional band appears at $1275 \mathrm{~cm}^{-1}$. Moreover, only one of the two bands $\left(\sim 1540 \mathrm{~cm}^{-1}\right)$ corresponding to asymmetric $\mathrm{NO}_{2}$ stretching mode is surface enhanced. It is particularly interesting the appearance of a new band at $717 \mathrm{~cm}^{-1}$ in the SERS spectrum of TNT which is absent in its normal Raman spectrum. This mode is associated to an asymmetric CCC in-plane ring bending deformation [30]. Comparison of the intensity of the other weak or nonsurface-enhanced Raman bands with the new band indicates a very selective chemical SERS enhancement as result of a very strong interaction of TNT with gold nanoparticles. These observations suggest a change in the molecular symmetry of TNT when it is adsorbed on the substrate. The enhancement of the in-plane deformation mode implies that TNT molecules lie perpendicular on the gold nanoparticles surface [27].

Normal Raman spectrum of 2,4-DNT is characterized by a very strong peak at $1352 \mathrm{~cm}^{-1}$ corresponding to $\mathrm{NO}_{2}$ symmetric stretching mode, two bands at 1544 and $1609 \mathrm{~cm}^{-1}$ assigned to asymmetric $\mathrm{NO}_{2}$ stretching and aromatic $\mathrm{NO}_{2}$ conjugation, respectively. The vibration mode assigned to the $\mathrm{H}-\mathrm{C}-\mathrm{C}$ in plane bending movement appears at $1203 \mathrm{~cm}^{-1}$, while the band at $1130 \mathrm{~cm}^{-1}$ is assigned to methyl $\mathrm{H}-\mathrm{C}-\mathrm{H}$ asymmetric bend. The other bands at 821 and $789 \mathrm{~cm}^{-1}$ 


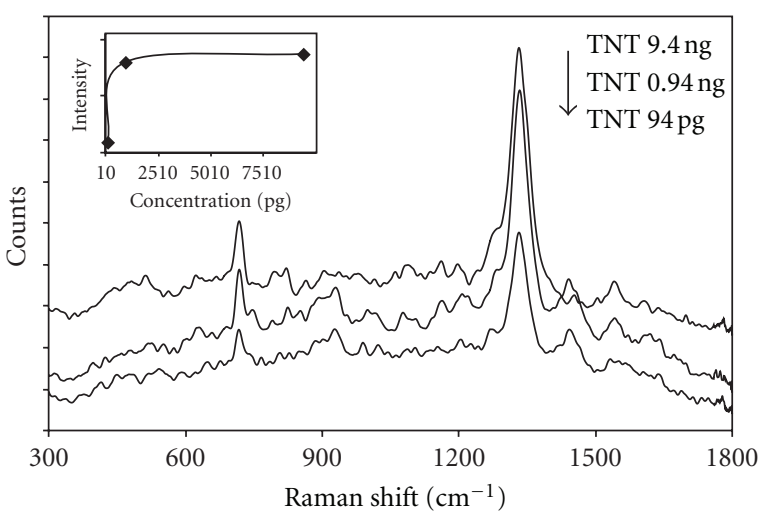

(a)

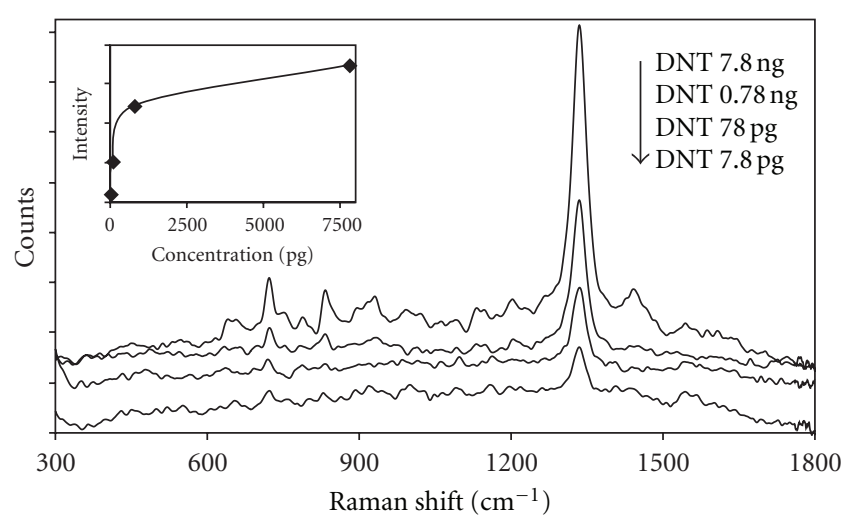

(b)

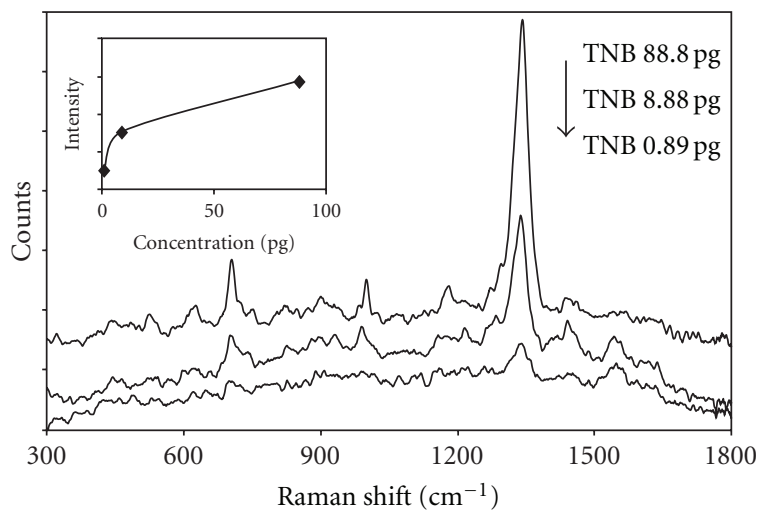

(c)

FIGURE 6: SERS spectra of (a) TNT, (b) 2,4-DNT, and (c) 1,3,5-TNB at different concentrations. Insets: variation of SERS intensity of NO stretching vibration with concentration.

correspond to out-of-plane $\mathrm{NO}_{2}$ mode and methyl rock vibrations, respectively.

SERS spectrum of 2,4-DNT shows bands corresponding to $\mathrm{NO}_{2}$ symmetric stretching vibration shifted to $1333 \mathrm{~cm}^{-1}$, while asymmetric stretching vibration was not SERS enhanced. All other bands appear to be SERS enhanced in minor proportion, although a new band at $717 \mathrm{~cm}^{-1}$ becomes visible as it happened with TNT molecule (Figure 6(b)).

The most significant modes of $1,3,5-\mathrm{TNB}$ are those assigned to symmetric (1364 and $1348 \mathrm{~cm}^{-1}$ ) and asymmetric $\left(1544 \mathrm{~cm}^{-1}\right) \mathrm{NO}_{2}$ vibrations, symmetric $\mathrm{CN}$ stretching mode observed to $1187 \mathrm{~cm}^{-1}$, ring breathing mode at $1006 \mathrm{~cm}^{-1}$, and $\mathrm{NO}_{2}$ symmetric deformation mode observed at $828 \mathrm{~cm}^{-1}$. It is possible to observe two different $\mathrm{NO}_{2}$ symmetric stretching vibrations for this compound, which has been previously reported for disubstituted nitroaromatics in solution [31,32]. Epstein et al. [31] have suggested that coupling of a vibrational mode of the $\mathrm{NO}_{2}$ group with a ring vibration with $\mathrm{CN}$ contribution is responsible for the lower wavenumber location band. The more electron-donating the second substituent on the ring, the smaller the frequency difference between the two $\mathrm{NO}_{2}$ vibrations.

SERS spectrum of TNB shows that most bands are surface enhanced except those near 1540 and $1650 \mathrm{~cm}^{-1}$
(Figure 6(c)). Spectroscopic differences between normal Raman and SERS spectra of TNB are the observation of just one $\mathrm{NO}_{2}$ symmetric stretching vibration at $1340 \mathrm{~cm}^{-1}$ rather than two, and the appearance of a new mode near $704 \mathrm{~cm}^{-1}$, as observed for SERS spectra of TNT and 2,4-DNT molecules.

The inset in Figure 6 shows the relationship between analyte concentration and the intensity of the SERS signals of the $\mathrm{NO}_{2}$ symmetric stretching vibration (in the range of $1330-1340 \mathrm{~cm}^{-1}$ ) for each analyte. Five microliters of analyte solution in ethanol were transferred to the substrates, and the solvent was allowed to evaporate at room temperature. Then, the SERS spectra were collected as soon as the analyte solution was dried. It is shown that the peak intensity increases in a nonlinear fashion with the concentration of each species, probably due to full coverage of explosives molecules onto high enhancement sites (hot spot). However, these nonlinear relationships become linear when a reciprocal style plot $(1 /[\mathrm{I}]$ versus $1 /[\mathrm{C}])$ of the SERS signal at $1330-1340 \mathrm{~cm}^{-1}$ was represented (Figure 7).

Considering that the laser spot has a diameter off about of $100 \mu \mathrm{m}$, the estimated low limit of detection (LOD) under the area illuminated by laser was $94 \mathrm{pg}$ of TNT, $7.8 \mathrm{pg}$ of $2,4-\mathrm{DNT}$, and $0.89 \mathrm{pg}$ of TNB. This clearly suggests that our flexible SERS-active substrate based in paper filter 


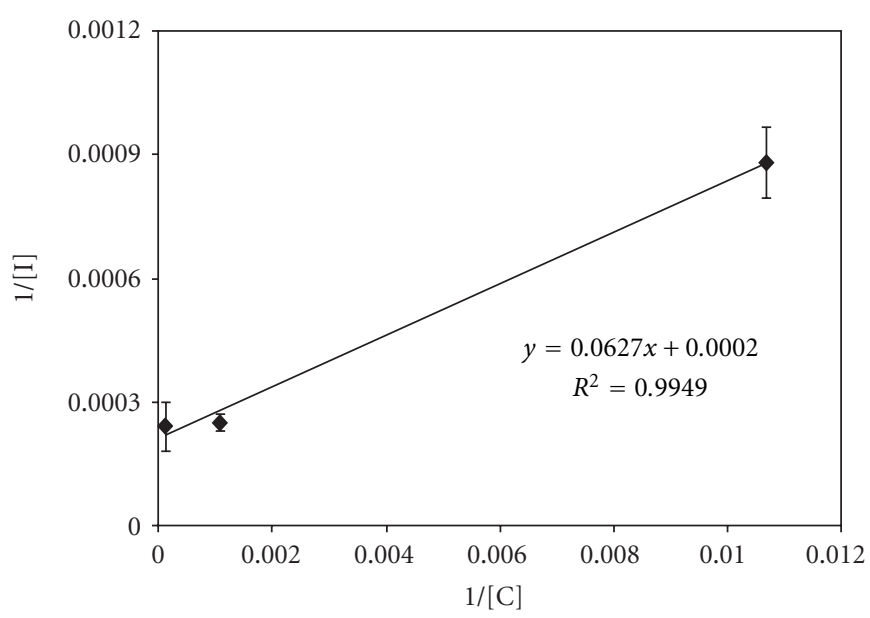

(a)

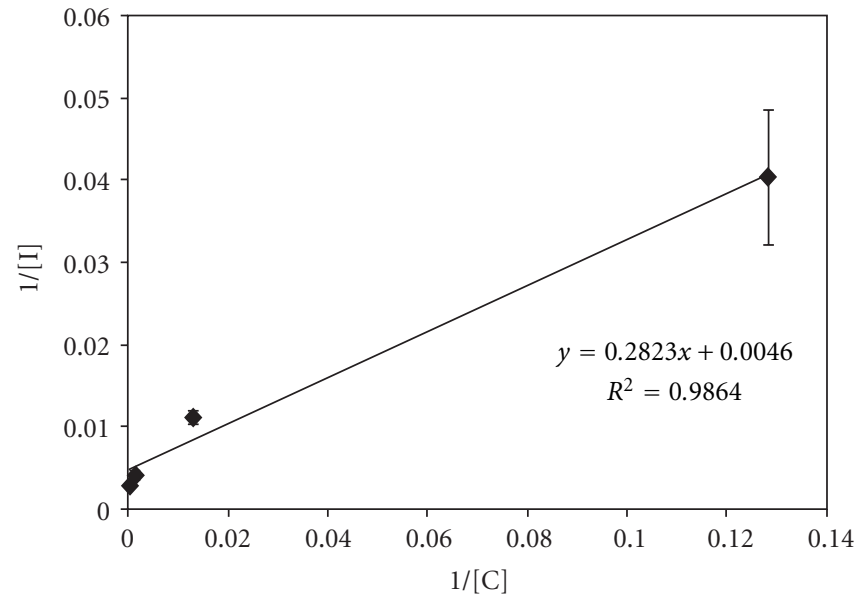

(b)

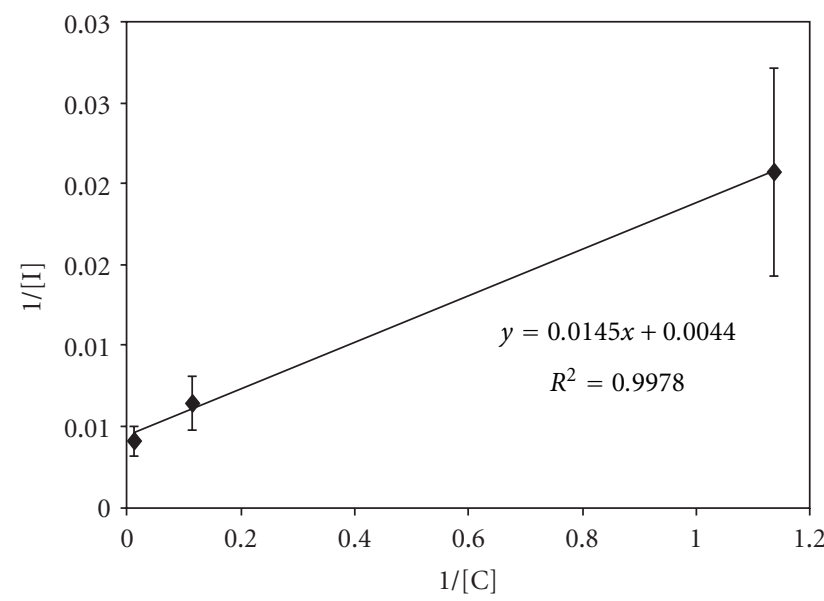

(c)

FIgURE 7: Plot of reciprocal of the $1330 \mathrm{~cm}^{-1}$ band intensity versus concentration for (a) TNT, (b) 2,4-DNT, and (c) 1,3,5-TNB.

Au-coated can be used to detect nitroaromatic HEM down to a subnanogram regime.

\section{Conclusion}

SERS experiments were performed on highly energetic materials molecules using a sensing platform fabricated by depositing gold nanoparticles on common lab filter paper (Whatman Grade 1) using thermal inkjet technology. The choice of paper as substrate for depositing plasmonic structures useful as possible SERS sensor brings numerous advantages such as flexibility, high specific surface area for an efficient uptake and transport of analyte to boundaries of metal nanostructures, extremely low cost, and simplicity of fabrication. The methodology presented an excellent performance, comparable to more elaborate techniques such as nanolithography or MFON. All these traits make the paper-based SERS substrates robust and friendly for numerous applications in routine lab uses or in situ to detect and identify several target analytes. The process described here can be easily scale up for batch fabrication.

\section{Acknowledgments}

Support from the US Department of Homeland Security under Award no. 2008-ST-061-ED0001 is acknowledged. However, the views and conclusions contained in this document are those of the authors and should not be interpreted as necessarily representing the official policies of the US Department of Homeland Security, neither expressed nor implied. The authors also acknowledge support from Hewlett-Packard Corporation in Aguadilla, P. R. Dr. Wilfredo Otaño, Professor of Physics from University of Puerto Rico Cayey, is acknowledged for facilitating the SEM images. This contribution was supported by the US Department of Defense, Proposal no. 58949-PH-REP, Agreement no. W911NF-11-1-0152. The authors also acknowledge contributions from Dr. Richard T. Hammond from Army Research Office, DoD.

\section{References}

[1] O. A. Sadik, A. K. Wanekaya, and S. Andreescu, "Advances in analytical technologies for environmental protection and 
public safety," Journal of Environmental Monitoring, vol. 6, no. 6, pp. 513-522, 2004.

[2] R. S. Das and Y. K. Agrawal, "Raman spectroscopy: recent advancements, techniques and applications," Vibrational Spectroscopy, vol. 57, no. 2, pp. 163-176, 2011.

[3] E. L. Izake, "Forensic and homeland security applications of modern portable Raman spectroscopy," Forensic Science International, vol. 202, no. 1-3, pp. 1-8, 2010.

[4] J. R. Baena and B. Lendl, "Raman spectroscopy in chemical bioanalysis," Current Opinion in Chemical Biology, vol. 8, no. 5, pp. 534-539, 2004.

[5] J. Jehlička, P. Vítek, H. G. M. Edwards, M. Heagraves, and T. Čapoun, "Application of portable Raman instruments for fast and non-destructive detection of minerals on outcrops," Spectrochimica Acta A, vol. 73, no. 3, pp. 410-419, 2009.

[6] M. Fleischmann, P. J. Hendra, and A. J. McQuillan, "Raman spectra of pyridine adsorbed at a silver electrode," Chemical Physics Letters, vol. 26, no. 2, pp. 163-166, 1974.

[7] M. E. Hankus, H. Li, G. J. Gibson, and B. M. Cullum, "Surface-enhanced Raman scattering-based nanoprobe for high-resolution, non-scanning chemical imaging," Analytical Chemistry, vol. 78, no. 21, pp. 7535-7546, 2006.

[8] S. Efrima and L. Zeiri, "Understanding SERS of bacteria," Journal of Raman Spectroscopy, vol. 40, no. 3, pp. 277-288, 2009.

[9] R. M. Jarvis and R. Goodacre, "Characterisation and identification of bacteria using SERS," Chemical Society Reviews, vol. 37, no. 5, pp. 931-936, 2008.

[10] R. A. Tripp, R. A. Dluhy, and Y. Zhao, "Novel nanostructures for SERS biosensing," Nano Today, vol. 3, no. 3-4, pp. 31-37, 2008.

[11] J. Guicheteau, L. Argue, D. Emge, A. Hyre, M. Jacobson, and S. Christesen, "Bacillus spore classification via surfaceenhanced Raman spectroscopy and principal component analysis," Applied Spectroscopy, vol. 62, no. 3, pp. 267-272, 2008.

[12] S. Botti, L. Cantarini, and A. Palucci, "Surface-enhanced Raman spectroscopy for trace-level detection of explosives," Journal of Raman Spectroscopy, vol. 41, no. 8, pp. 866-869, 2010.

[13] N. A. Hatab, G. Eres, P. B. Hatzinger, and B. Gu, "Detection and analysis of cyclotrimethylenetrinitramine (RDX) in environmental samples by surface-enhanced Raman spectroscopy," Journal of Raman Spectroscopy, vol. 41, no. 10, pp. 1131-1136, 2010.

[14] W. E. Doering and S. Nie, "Single-molecule and singlenanoparticle SERS: examining the roles of surface active sites and chemical enhancement," Journal of Physical Chemistry B, vol. 106, no. 2, pp. 311-317, 2002.

[15] M. Fan and A. G. Brolo, "Silver nanoparticles self assembly as SERS substrates with near single molecule detection limit," Physical Chemistry Chemical Physics, vol. 11, no. 34, pp. 73817389, 2009.

[16] P. C. Lee and D. Meisel, "Adsorption and surface-enhanced Raman of dyes on silver and gold sols," Journal of Physical Chemistry, vol. 86, no. 17, pp. 3391-3395, 1982.

[17] M. A. De Jesús, K. S. Giesfeldt, J. M. Oran, N. A. AbuHatab, N. V. Lavrik, and M. J. Sepaniak, "Nanofabrication of densely packed metal-polymer arrays for surface-enhanced raman spectrometry," Applied Spectroscopy, vol. 59, no. 12, pp. 1501-1508, 2005.
[18] Y. Song and H. E. Elsayed-Ali, "Aqueous phase Ag nanoparticles with controlled shapes fabricated by a modified nanosphere lithography and their optical properties," Applied Surface Science, vol. 256, no. 20, pp. 5961-5967, 2010.

[19] U. S. Dinish, F. C. Yaw, A. Agarwal, and M. Olivo, "Development of highly reproducible nanogap SERS substrates: comparative performance analysis and its application for glucose sensing," Biosensors and Bioelectronics, vol. 26, no. 5, pp. 1987-1992, 2011.

[20] L. A. Dick, A. D. McFarland, C. L. Haynes, and R. P. Van Duyne, "Metal film over nanosphere (MFON) electrodes for surface-enhanced Raman spectroscopy (SERS): improvements in surface nanostructure stability and suppression of irreversible loss," Journal of Physical Chemistry B, vol. 106, no. 4, pp. 853-860, 2002.

[21] P. L. Stiles, J. A. Dieringer, N. C. Shah, and R. P. Van Duyne, "Surface-enhanced Raman spectroscopy," Annual Review of Analytical Chemistry, vol. 1, no. 1, pp. 601-626, 2008.

[22] W. W. Yu and I. M. White, "Inkjet printed surface enhanced raman spectroscopy array on cellulose paper," Analytical Chemistry, vol. 82, no. 23, pp. 9626-9630, 2010.

[23] C. H. Lee, L. Tian, and S. Singamaneni, "Paper-based SERS swab for rapid trace detection on real-world surfaces," ACS Applied Materials and Interfaces, vol. 2, no. 12, pp. 3429-3435, 2010.

[24] C. H. Lee, M. E. Hankus, L. Tian, P. M. Pellegrino, and S. Singamaneni, "Highly sensitive surface enhanced Raman scattering substrates based on filter paper loaded with plasmonic nanostructures," Analytical Chemistry, vol. 83, no. 23, pp. 8953-8958, 2011.

[25] Y. H. Lee, D. W. Kim, S. I. Shin, and S. G. Oh, "Preparation of Au colloids by polyol process using $\mathrm{NaHCO}_{3}$ as a buffering agent," Materials Chemistry and Physics, vol. 100, no. 1, pp. 85-91, 2006.

[26] J. Akhavan, The Chemistry of Explosives, The Royal Society of Chemistry, 2nd edition, 2004.

[27] J. I. Jerez-Rozo, O. M. Primera-Pedrozo, M. A. Barreto-Cabán, and S. P. Hernández-Rivera, "Enhanced Raman scattering of 2,4,6-TNT using metallic colloids," IEEE Sensors Journal, vol. 8, no. 6, pp. 974-982, 2008.

[28] K. Kneipp, Y. Wang, R. R. Dasari et al., "Near-infrared surfaceenhanced Raman scattering of trinitrotoluene on colloidal gold and silver," Spectrochimica Acta A, vol. 51, no. 12, pp. 2171-2175, 1995.

[29] J. Clarkson, W. E. Smith, D. N. Batchelder, D. A. Smith, and A. M. Coats, "A theoretical study of the structure and vibrations of 2,4,6-trinitrotolune," Journal of Molecular Structure, vol. 648, no. 3, pp. 203-214, 2003.

[30] S. P. Hernández-Rivera and J. Castillo-Chará, "Ab initio, DFT calculation and vibrational analysis of 2,4,6- trinitrotoluene," Vibrational Spectroscopy, vol. 53, no. 2, pp. 248-259, 2010.

[31] L. M. Epstein, E. S. Shubina, L. D. Ashkinadze, and L. A. Kazitsyna, "Nitro group vibrations in spectra of nitroaromatic compounds with donor substituents," Spectrochimica Acta A, vol. 38, no. 3, pp. 317-322, 1982.

[32] J. F. Bertrán, M. Hernández, and B. La Serna, "Study of the anomalous $\mathrm{NO}_{2}$ symmetric stretch in $p-\mathrm{NO}_{2}$ aniline," Spectrochimica Acta A, vol. 38, no. 2, pp. 149-151, 1982. 


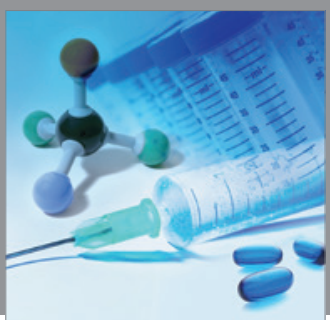

International Journal of

Medicinal Chemistry

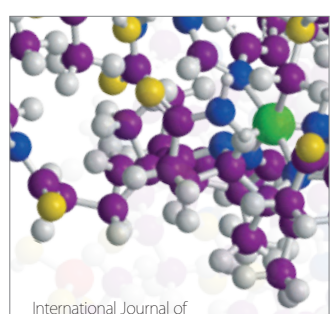

Carbohydrate Chemistry

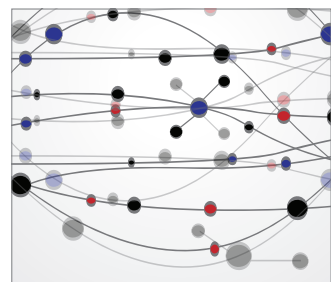

The Scientific World Journal
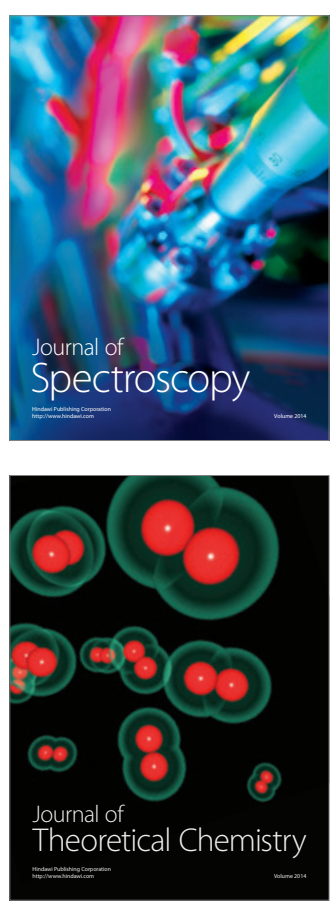
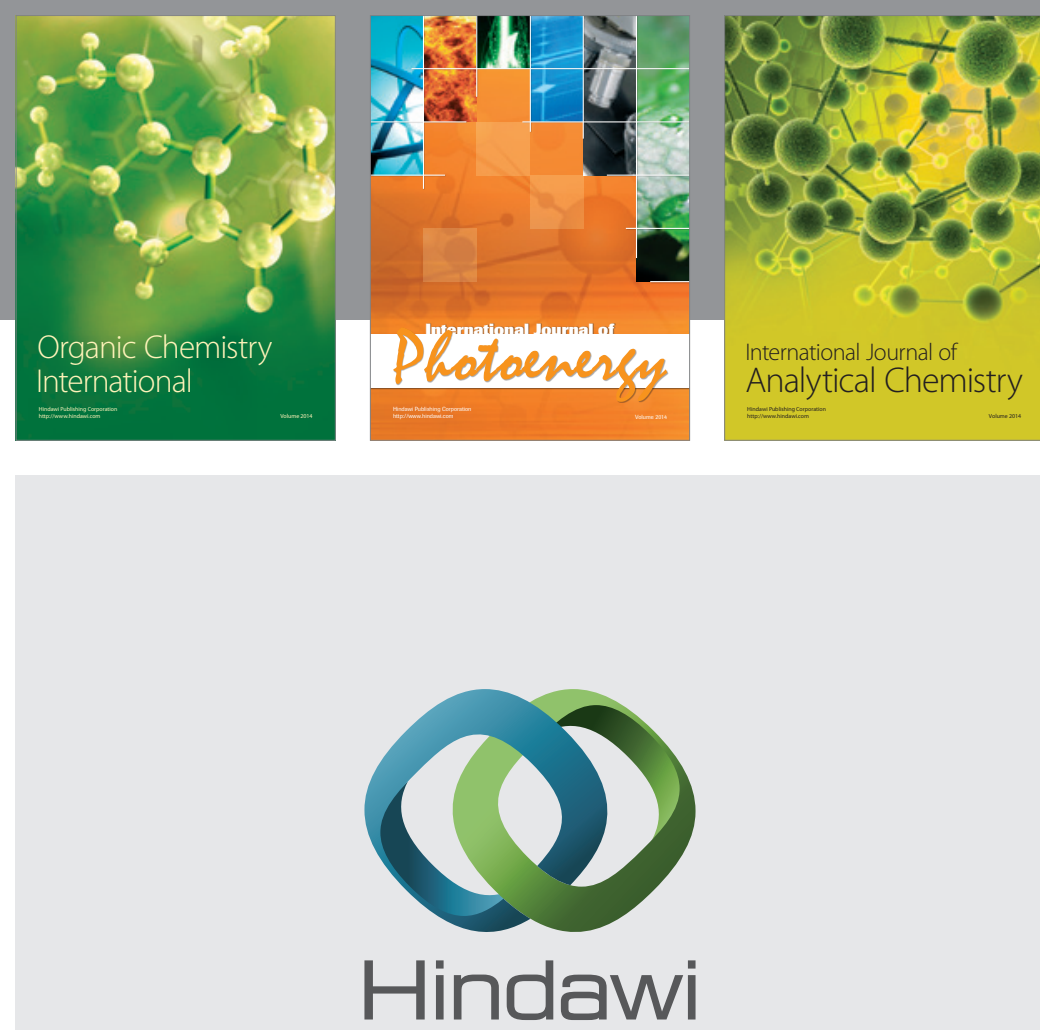

Submit your manuscripts at

http://www.hindawi.com
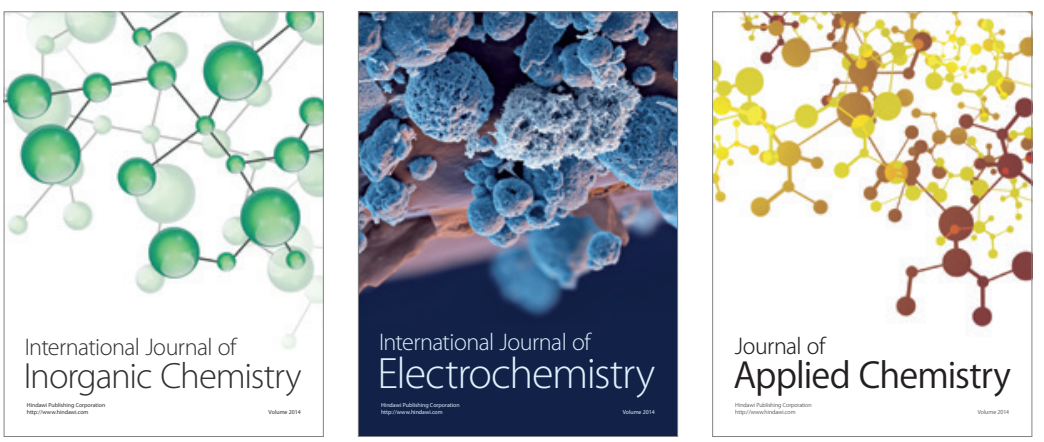

Journal of

Applied Chemistry
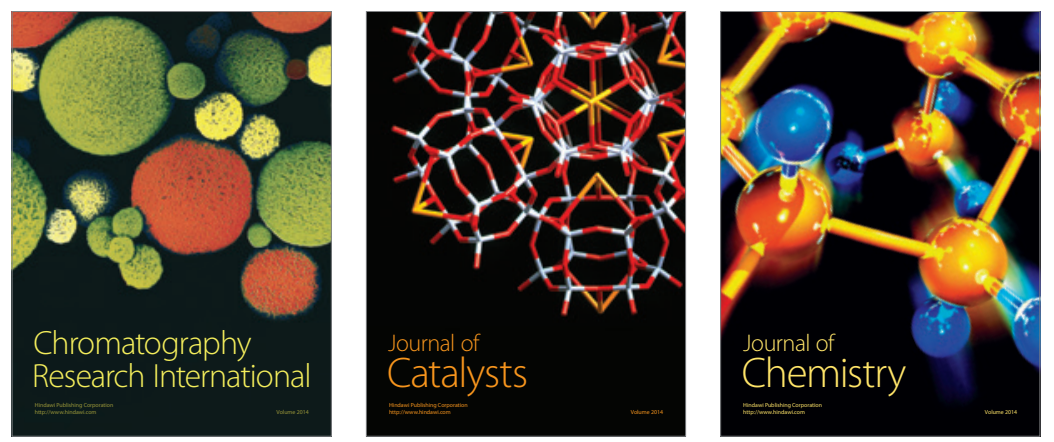
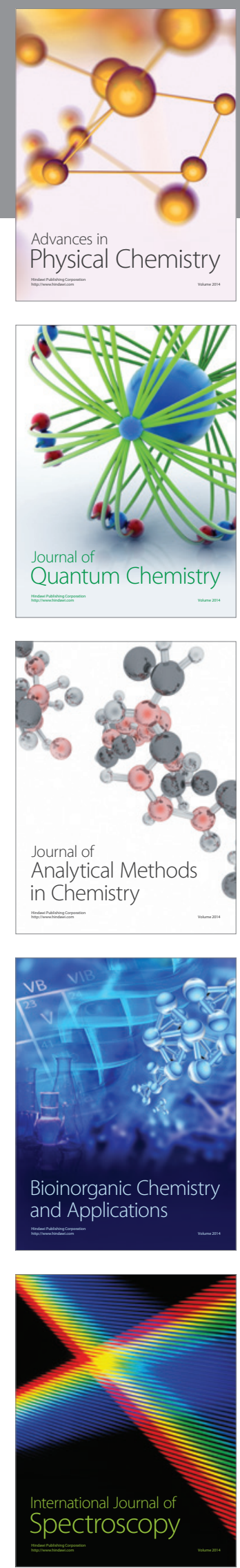